\title{
Diacronie
}

Studi di Storia Contemporanea

$\mathrm{N}^{\circ} 29,1 \mid 2017$

"Crash test"

\section{La Icaria di Étienne Cabet: un'utopia letteraria del XIX secolo}

José D'Assunção Barros

Traduttore: Jacopo Bassi

\section{OpenEdition}

Journals

Edizione digitale

URL: http://journals.openedition.org/diacronie/4947

DOI: 10.4000/diacronie.4947

ISSN: 2038-0925

Editore

Association culturelle Diacronie

\section{Notizia bibliografica digitale}

José D'Assunção Barros, «La Icaria di Étienne Cabet: un'utopia letteraria del XIX secolo », Diacronie

[Online], $N^{\circ}$ 29, 1 | 2017, documento 1, Messo online il 29 mars 2017, consultato il 22 avril 2019. URL : http://journals.openedition.org/diacronie/4947 ; DOI : 10.4000/diacronie.4947 


\title{
Diacronie
}

Studi di Storia Contemporanea

29, 1/2017

"Crash test". Continuità, discontinuità, legami e rotture nelle dinamiche della storia contemporanea

\section{La Icaria di Étienne Cabet: un'utopia letteraria del XIX secolo}

\author{
José D’Assunção BARROS
}

Per citare questo articolo:

BARROS, José D’Assunção, «La Icaria di Étienne Cabet: un'utopia letteraria del XIX secolo», Diacronie. Studi di Storia Contemporanea : "Crash test". Continuità, discontinuità, legami e rotture nelle dinamiche della storia contemporanea, 29, $1 / 2017,29 / 3 / 2017$,

URL: < http://www.studistorici.com/2017/03/29/barros_numero_29/ >

Diacronie Studi di Storia Contemporanea $\rightarrow$ http://www.diacronie.it Rivista storica online. Uscita trimestrale.

redazione.diacronie@hotmail.it

Comitato di direzione: Naor Ben-Yehoyada - João Fábio Bertonha - Christopher Denis-Delacour - Maximiliano Fuentes Codera Anders Granås Kjøstvedt - John Paul Newman - Deborah Paci - Niccolò Pianciola - Spyridon Ploumidis - Wilko Graf Von Hardenberg

Comitato di redazione: Jacopo Bassi - Luca Bufarale - Gianluca Canè - Fausto Pietrancosta - Alessandro Salvador - Matteo Tomasoni Diritti: gli articoli di Diacronie. Studi di Storia Contemporanea sono pubblicati sotto licenza Creative Commons 3.0. Possono essere

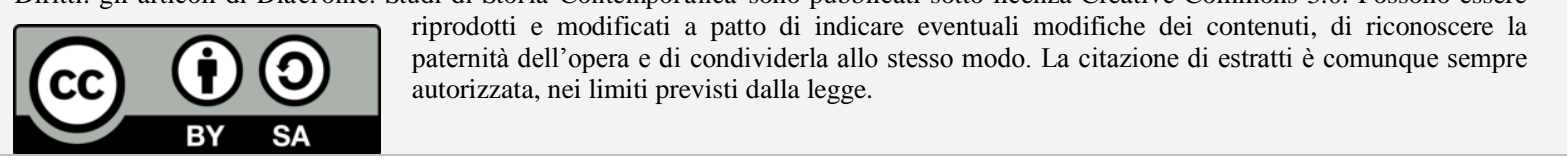




\title{
1/ La Icaria di Étienne Cabet: un'utopia letteraria del XIX secolo
}

José D’Assunção BARROS

Traduzione di Jacopo BASSI

\begin{abstract}
Questo articolo prende in analisi la letteratura utopica del XIX secolo, soffermandosi in particolare sulle società immaginarie ideate da Étienne Cabet, nel romanzo intitolato Voyage en Icarie (1842). Questa analisi più specifica è preceduta da una breve introduzione alla letteratura utopica a partire dal XVI secolo - recuperando in chiave comparativa proposte di autori come Tommaso Moro, Campanella, Francis Bacon, Fourier, Saint-Simon e Robert Owen - e oltre ad una discussione teorica sui modelli di distribuzione dell'uguaglianza, in questo caso prendendo in considerazione le riflessioni di Norberto Bobbio e Amartya Sen su questo tema. L'opera di Cabet è affronta ta nel suo contesto, e questo si giustifica in grazia del fatto che seppe gettare un ponte tra letteratura e società, in quanto questi lavori suscitarono grande interesse tra i lettori del tempo e ispirarono la realizzazione di specifiche esperienze utopiche.
\end{abstract}

\section{Prefazione}

Lo sforzo nel conciliare l'idea di uguaglianza con la constatazione delle differenze tra gli uomini ha costituito un sogno antico come la civilizzazione. La sfida insita nel pensare sistemi in cui sia possibile realizzare l'uguaglianza, solo in alcuni ambiti o anche a tutti i livelli, sorse tanto tra i rivoluzionari e i riformatori che si impegnarono nella costruzione e ricostruzione di società reali, quanto tra gli artisti e i letterati che idealizzarono quelle immaginarie. Spesso, del resto, la letteratura e l'arte hanno influenzato la realtà e gli echi di un'immaginazione utopica espressi in prima battuta sotto forma di romanzi e opere artistiche sono passate dalle pagine di un libro ad essere concretizzate. In questo articolo il nostro obiettivo è quello di riflettere su di un autore ottocentesco che ideò - in un particolare romanzo - una società utopica divenuta modello per la realizzazione concreta di un'esperienza umana egualitaria. In un secolo nel quale non furono rare le elaborazioni associate all'immaginazione utopica, il politico socialista Étienne Cabet (1788-1856), in 
un romanzo intitolato Voyage en Icarie (1840), concretizzò l'ideale di una società perfetta sistematizzando il tema dell'uguaglianza. Politico e militante socialista, coinvolto nei movimenti sociali del suo tempo e interessato a trasformare la realtà concreta, Cabet passò dall'immaginazione utopica alla pratica utopista, andando a ingrossare le fila di coloro che tentarono di costruire effettive comunità socialiste.

Nelle prossime pagine ci proponiamo, più nello specifico, di verificare come Cabet affrontò le idee di uguaglianza, disuguaglianza e differenza nel suo romanzo utopico, di come pensò un sistema sociale e idealizzò uno spazio di convivenza, oltre a presentare la relazione tra produzione e lavoro e tra queste due e l'ozio, e di come l'opera concili la necessità di governo con la categoria della libertà. Questioni come queste - che sono quasi sempre presenti nelle utopie immaginate sinora e che acquisiscono una loro specificità nel romanzo utopico di Cabet - divengono importanti nella sua produzione letteraria perché sono reali, concrete nelle società in cui i loro autori vissero, così come nelle società in cui viviamo odiernamente.

\section{Utopie}

La definizione di "utopia" porta con sé una storia vasta e complessa, risale risalente alla prima utilizzazione del termine da parte di Tommaso Moro (1516) e che si avvale delle molteplici ridefinizioni posteriori, siano state esse positive o negative ${ }^{1}$. Per Moro, scrittore rinascimentale inglese che ha il merito di avere introdotto il termine nello scenario letterario e politico, l'espressione si rifaceva, per omofonia, al contempo al "non-luogo" di una comunità situata al di fuori del nostro spazio e della nostra linea del tempo (ou-topia) e al "luogo della felicità" consolidato attraverso una società perfetta società perfetta (eu-topia) ${ }^{2}$. Questa sovrapposizione di sensi - il "nonluogo geografico" nel quale si stabilisce un "paese immaginario" e il "luogo della felicità" nel quale si ridisegna una società perfetta - sarà presente in diverse delle realizzazioni letterarie dell'immaginazione utopica. Impiegheremo il termine in questa doppia accezione, per via del fatto

\footnotetext{
${ }^{1}$ Uno studio di questa storia, in lingua francese, è stato realizzato da FUNKE, Hans Gunter. L'évolution sémantique de La notion d'Utopie en français, in HUDDE, Heinrich, KUON, Peter (eds.), De l'utopie a l'uchronie. Formes, Significations, Fonctions, Tübingen, Gunther Narr Verlag, 1988, pp. 19-37. Una collettanea con diversi saggi sul tema dell'utopia è stata curata da: NEUSÜSS, Arnhelm (ed.), Utopia, Barcelona, Barral, 1971; si veda anche SUVIN, Darko, Pour une définition de l'utopie comme genre littéraire, in ID., Pour une poétique de la sciencefiction. Études en théorie et en histoire d'un genre littéraire, Montréal, Les Presses de l'Université du Quebéc, 1977, pp. 47-69.

${ }^{2}$ Su questo si veda: TROUSSON, Raymond, «Utopia e utopismo», in Morus. Utopia e Renascimento, 2, 2005, pp. 123-135, p. 125. Il lettore italiano troverà il testo di Trousson da cui è ricavato l'articolo in portoghese in MINERVA, Nadia, Per una definizione dell'utopia: metodologie e discipline a confronto. Atti del Convegno internazionale di Bagni di Lucca, 12-14 settembre 1990, Ravenna, Longo editore, 1992.
} 
che questi due significati risultano in sintonia nell'opera Voyage en Icarie, su cui ci soffermeremo in seguito ${ }^{3}$.

L'Icarie di Cabet, inoltre, si rifà a un "viaggio immaginario",significato principale che la parola utopia aveva nella lingua francese del XVII secolo ${ }^{4}$. Impiegheremo comunque la parola, pur coscienti di un altro possibile senso, oltre a quello svalutativo che è stato attribuito al lemma nel XIX secolo, sia dalla critica borghese, sia da quella marxista. Si tratta dell'idea, proposta da Karl Mannheim ${ }^{5}$, per cui l'utopia rappresenterebbe un pensiero che contesta radicalmente il sistema in vigore, così da renderla una speranza ${ }^{6}$. Relativamente a questo contenuto semantico, possiamo correlarla anche con l'invenzione letteraria elaborata da Cabet. Producendo una visione radicalmente opposta alla realtà esistente, considerando la prospettiva proposta da Bronislaw Baczko ${ }^{7}$, le utopie si mostrano come «radicalmente critiche». Queste custodiscono, in un modo o nell'altro, la volontà di modificare il corso della storia, vengono caricate dall'intenzione di trasformare il mondo e per questa ragione operano sul piano della veridicità: ossia a partire dalla proposta di un modello realizzabile, anche se non si dimostra come sarebbe possibile metterlo in pratica.

Tornando al mondo delle creazioni letterarie che orientano visioni radicalmente opposte ai sistemi vigenti con l'intento di operare una loro critica radicale, è oggi possibile parlare di un'immaginazione utopica o di un genere utopico che già vanta una lunga tradizione storica. Inoltre, in momenti diversi sono già state designate come utopie i progetti di autori che desideravano realizzare una nuova società, come Charles Fourier o Henri de Saint-Simon, o che diedero vita persino a esperienze concrete con l'intento di stabilire comunità ideali o colonie socialiste, come Robert Owen ${ }^{8}$. Questi tre tipi di possibilità corrispondono alla «gamma completa degli studi utopici: finzioni, oggetti e colonie» ${ }^{9}$. Con il passaggio dalle finzioni utopiche ai progetti e alle colonie che portarono alla creazione effettiva di una società nuova, passiamo dal genere utopico propriamente

\footnotetext{
${ }^{3}$ Se è impossibile pervenire ad una definizione consensuale di utopia - quantomeno per la categoria letteraria del "genere utopico" che andremo a discutere - possiamo riconoscere come esistano una serie di definizioni, succedutesi nel tempo, che rispondono alle specificità dei vari autori e delle questioni della loro epoca. Cfr.: LEVITAS, Ruth, The Concept of Utopia, Syracuse, Syracuse University Press, 1990; QUARTA, Cosimo, «Utopia: gênese de uma palavra-chave», in Morus. Utopia e Renascimento, 3, 2006, pp. 35-53.

${ }^{4}$ TROUSSON, Raymond, op. cit., p. 126.

${ }^{5}$ MANNHEIM, Karl, Ideologie und Utopie, Bonn, Verlag Fr. Cohen, 1929 [trad. it. Ideologia e utopia, Bologna, Il Mulino, 1985].

${ }^{6}$ BLOCH, Ernst, O Princípio da Esperança, Rio de Janeiro, EdUERJ, 2005 [trad. it. Il principio speranza, Milano, Garzanti, 2005].

${ }^{7}$ BACZKO, Bronislaw, Orwell e Solzenicyn: far fronte al totalitarismo, in MINERVA, Nadia, op. cit., p. 173-184, p. 173.

${ }^{8}$ I progetti utopisti come quelli di Fourier e Saint-Simon - nei quali troviamo un «orizzonte isolato, senza un luogo utopico» - vengono considerati da Darko Suvin, per la loro tipologia, come un "pensiero utopico non localizzato». SUVIN, Darko «Um breve tratado sobre a Distopia 2001», in Morus. Utopia e Renascimento, 10, 2015, pp. 465-488. Da queste possiamo distinguere esperienze come quella di Robert Owen, che trovò una sua collocazione, cessando di essere un progetto per concretizzarsi come una realizzazione propriamente detta. Le finzioni (il «genere utopico»), non comportavano l'impegno derivante dal proporsi come progetti e agivano principalmente come strumenti critici della realtà con cui si confrontavano. Questo fu il caso di Utopia di Tommaso Moro, ma anche della Icaria fittizia di Étienne Cabet.

${ }^{9}$ SUVIN, Darko, «Um breve tratado sobre a Distopia 2001», cit., p. 472.
} 
detto ai progetti e alle realizzazioni utopiste, due campi che successivamente definiremo meglio. Tra questi due poli - quello dell'immaginazione utopica e quello dei progetti e delle realizzazioni utopiste - troviamo autori peculiari che si muovevano e si collocavano tra l'immaginazione utopica espressa in un'opera letteraria e la realizzazione utopista proposta attraverso l'instaurazione concreta di una comunità ideale, come ad esempio lo stesso Cabet, la cui immaginazione utopica verrà esaminata in questo articolo a partire dalla sua opera letteraria Voyage en Icarie (1840).

Osserviamo come fino a qui la nozione di utopia, oggetto di molteplici definizioni e controversie, permetta di accorpare - pur con tutte le limitazioni del caso - opere molto diverse che possono essere divise in tre grandi categorie, a seconda della loro ipoteticità, progettabilità o realizzazione pratica. Anche senza elaborare interpretazioni più precise e suddivisioni possibili del campo utopico, possiamo richiamare in via preventiva una definizione più ampia che abbracci tutti i casi:

L'Utopia sarà definita come la costruzione di una particolare comunità dove le istituzioni politiche, le norme e le relazioni tra le persone sono organizzate in accordo con un principio radicalmente differente da quello della comunità dell'autore: questa costruzione è basata sull'estraniamento [estrangement] prodotto da un'ipotesi storica alternativa; è creata da classi sociali interessate all'alternativa e al cambiamento ${ }^{10}$.

Per parte nostra resteremo coscienti dei limiti imposti dalla definizione allargata di utopia, pur senza vietarci l'impiego del termine. Riconosceremo, anzitutto, che non esiste propriamente una linea di continuità tra le utopie e, più nello specifico, che leghi gli utopisti del XIX secolo gli uni con gli altri ${ }^{11}$. Il raggruppamento in un unico campo di autori che sarebbero successivamente stati considerati utopisti fu il risultato di una grande costruzione intellettuale a cui contribuirono linee di analisi differenti. Una lettura d'insieme di autori oggi considerati come utopici si verifica, ad esempio, a partire dalla valutazione di Marx ed Engels delle prospettive socialiste espresse da tali autori utopici, che i due fondatori del materialismo consideravano non rientranti nella categoria da loro elaborata di «socialismo scientifico» ${ }^{12}$. Fermo restando ciò - il riconoscimento delle discontinuità e delle differenze tra i diversi pensatori che vennero definiti "utopici" - possiamo d'altro canto riconoscere anche che esistono possibili intertestualità (autori legati al pensiero utopista o al genere letterario utopico che hanno letto altri autori della loro epoca e di altre epoche).

\footnotetext{
${ }^{10}$ Ibidem, p. 468. Un altro autore importante, Luigi Firpo sposa questa visione secondo cui un'Utopia necessita di essere globale, non riferendosi solamente a un aspetto della vita umana, ma alla sua totalità. FIRPO, Luigi, "Por uma definição da "Utopia"», in Morus. Utopia e Renascimento, 2, 2005, pp. 227-237, p.229.

${ }^{11}$ BACZKO, Bronislaw, «Utopia», in Enciclopédia Einaudi, vol. 5, Lisboa, Imprensa Nacional - Casa da Moeda, 1985, pp .333-396.

${ }^{12}$ ENGELS, Friedrich, Socialisme utopique et socialisme scientifique, Paris, Dervaux Libraire-Éditeur, 1880 [L'evoluzione del socialismo dall'utopia alla scienza, Napoli, Med. invest, 1992]. Per uno studio sulla relazione tra utopismo e marxismo, si veda GEOGHEGAN, Vincent, Utopianism and Marxism, London, Methuen, 1987.
} 
Questo significa che, se ogni costruzione utopica deve essere considerata nel suo contesto storico, è possibile anche considerarne l'insieme a partire dai problemi posti dal ricercatore, nell'ambito dei quali si stabiliscono connessioni e intertestualità.

Un modo interessante per fare chiarezza nell'ambito in cui lavoriamo è distinguere tra un peculiare modello narrativo che finì per costituirsi in quello che possiamo chiamare "genere utopico" (l'utopia come genere letterario) e i progetti sociopolitici o le realizzazioni più specifiche che possono essere associate a un pensiero utopista con la chiara intenzione e volontà di essere trasposte in pratica, come avvenne nel caso dei progetti di Fourier, Saint-Simon o di altri. Consideriamo infatti quelle di utopia e utopismo come nozioni che dialogano fra loro, ma che non necessariamente si sovrappongono ${ }^{13}$. Per ora rimaniamo nell'ambito delle creazioni letterarie, che è quello che attirerà maggiormente la nostra attenzione in questo articolo, notando preliminarmente come il primo iato che si presenta tra le utopie come genere letterario, da una parte, e i progetti di realizzazione utopista, dall'altro, è che le prime gettano un ponte verso il futuro, mentre i secondi mirano a concretizzarsi nel presente o quantomeno si confrontano con questa possibilità ${ }^{14}$.

A prescindere dalle differenze, esiste un "genere utopico" la cui storia può essere tracciata a partire da una serie di opere letterarie che sono tanto figlie delle società e delle circostanze in cui si creare sono state create, quanto delle intertestualità di cui hanno beneficiato ${ }^{15}$. Alcuni aspetti sono ricorrenti in molte delle creazioni letterarie che possiamo classificare all'interno del "genere utopico". Troviamo inizialmente l'impegno nel descrivere una società realizzabile per ciò che riguarda la struttura e passibile di essere ritenuta dal lettore come dotata di caratteristiche di verosimiglianza, al contrario delle creazioni letterarie del tutto chimeriche come la cuccagna medievale, che non prevede un sistema lavorativo e produttivo, ma un'abbondanza infinita che

\footnotetext{
${ }^{13}$ L'utopismo è una modalità dell'immaginario politico, spesso un programma radicale di trasformazione sociale della realtà esistente, ma più genericamente un «esercizio mentale sui possibili laterali», secondo la formula di Raymond Ruyer. RUYER, Raymond, L'Utopie et les utopies, Paris, PUF., 1950, p. 9. «L'utopia è un genere letterario che corrisponde alla codificazione della modalità utopica attraverso una forma scritta e, più precisamente, narrativa». RACAULT, Jean-Michel, «Da ideia de perfeição como elemento definidor da utopia: as utopias clássicas e a natureza humana», in Morus. Utopia e Renascimento, 6, 2009, pp. 29-45, p. 31. La divisione fra il "genere utopico» e una «modalità utopica» è stata proposta per la prima volta da Ruyer (RUYER, Raymond, op. cit.) e Cioranescu (CIORANESCU, Alexandre, L'avenir du passe: utopie et littérature, Paris, Gallimard, 1972) è stato il primo a utilizzare l'espressione «utopismo» per designare questa modalità utopica, all'interno delal quale il «genere utopico» si inquadra come un caso particolare. Si veda anche: QUARTA, Cosimo, «Homo utopicus. On the Need for Utopia», in Utopian studies, 7, 2/1996, pp. 153-166.

${ }^{14}$ Luigi Firpo, discutendo la coscienza che alcuni autori di utopia hanno del fatto che il loro messaggio possa maturare nel futuro, fa ricorso all'immagine per cui: «L'utopia [il genere utopico] è storicamente, un messaggio nella bottiglia, il messaggio di un naufrago». FIRPO, Luigi, op. cit., p. 230.

${ }^{15}$ «Se è vero che la coscienza utopica inventa o diversifica i suoi mezzi espressivi, non è meno vero che esiste una categoria di testi che agiscono secondo alcune costanti per via delle quali deve essere possibile riunirle in un genere più o meno coerente». TROUSSON, Raymond, op. cit., p. 127. Inoltre, secondo ciò che aggiunge Trusson: «Se tutto il testo letterario è al contempo il prodotto di una combinazione presistente e di una modificazione - più o meno profonda - di questa combinazione, questo testo completa ed estrapola al tempo stesso il genere senza, per questo motivo, rompere con esso». Ibidem, p.131.
} 
nasce spontaneamente dalla terra. Con questo vogliamo dire che le utopie sviluppano giocoforza una logica in grado di essere compresa e accetta dai suoi lettori assieme a tutta la loro stravaganza.

Anche se si posizionano al di fuori del genere utopico, tuttavia sono strumenti critici simili le società che si distaccano molto dalla condizione umana reale, come avviene nel caso dei celebri Gulliver's Travels ${ }^{16}$, dove si descrivono comunità costituite da gente minuscola, giganti o cavalli razionali. La letteratura utopica propriamente detta mette in scena uomini e donne normali, senza poteri speciali o caratteristiche sostanziali differenti da quelle incontrate nella realtà umana che si desidera criticare ${ }^{17}$. Gli abitanti delle utopie non sono divinità e neppure eroi, il che distingue questo genere dai miti, nei quali vengono descritte le età dell'oro, evidenziando anche come, mentre queste si riferiscono a qualcosa che si è perduto, talvolta irrimediabilmente, le utopie mirano sempre, attraverso l'orizzonte che ridisegnano, a qualcosa che si desidera conquistare ${ }^{18}$. Questa scelta permette di allontanare dal genere utopico anche costruzioni mitiche come quelle elaborate da Platone, compresa la sua Politeia ${ }^{19}$, una repubblica ideale che sarebbe esistita in un'Atlantide situata in un passato molto remoto ${ }^{20}$. Al passato distante, oramai perduto, le utopie letterarie preferiscono il presente alternativo di una realtà parallela ${ }^{21}$ oppure il futuro enigmatico che si pone come un orizzonte, come nel caso dell'America visitata a cento anni di distanza di Looking Backward 2000-188722.

Inoltre, le utopie letterarie (o) sono abitualmente caratterizzate dal viaggio. Il personaggio principale dell'opera di Tommaso Moro ha viaggiato per raggiungere la sua Utopia, benché questa si trovasse in un non luogo, così come i personaggi principali della Città del Sole di Campanella, della New Atlantis di Francis Bacon, o della Icarie di Cabet, giungono al loro destino utopico grazie a un percorso. Allo stesso modo Edward Bellamy (1850-1898), nel suo romanzo Looking Backward 2000-1887, fa sì che il suo personaggio principale raggiunga la sua società utopica attraverso un viaggio, per quanto questo si svolga nel tempo. In tutti questi casi, il percorso si rivela un elemento tipico delle

\footnotetext{
${ }^{16}$ SWIFT, Jonathan, Travels into Several Remote Nations of the World. In Four Parts. By Lemuel Gulliver, First a Surgeon, and then a Captain of Several Ships, London, Motte, 1726.

${ }^{17}$ Un'eccezione interessante è rappresentata dall'utopia creata dall'ex-monaco francescano Gabriel de Foigny: La terre australe connue (1676). Il paese immaginario da lui descritto è abitato da una razza umana pre-adamita di ermafroditi, i quali, per le loro peculiarità fisiche, si trovano al di sopra delle normali passioni umane. Per via del fatto di non essere discendenti di Adamo e, quindi, non essendo soggetti alle conseguenze della Caduta, questi abitanti utopici possiederebbero una natura umana più perfezionata. Partecipando tutti di una ragione perfetta, non necessitano neppure di leggi. In ogni modo, contrariamente a questo modello, le utopie che ricorrono più spesso devono spesso affrontare la sfida di pensare una società istituzionalmente perfetta per esseri umani nella loro condizione normale.

${ }^{18}$ Sui generi vicini e apparentabili al genere utopico, si veda: TROUSSON, Raymond, op. cit., pp. 128-130.

${ }^{19}$ PLATONE, La repubblica; PLATONE, Timeo.

${ }^{20}$ Questo aspetto è oggetto di un'eventuale discordanza tra gli autori che si sono specializzati nello studio delle utopie. Al contrario di Trousson (TROUSSON, Raymond, op. cit., p.128), che separa le età dell'Oro dal corpus utopico, Racault ammette che l'avvenire desiderato dall'utopismo possa essere caratterizzato dall'«aspetto paradossale di un ritorno a un passato più antico» (RACAULT, Jean-Michel, op. cit., p. 31).

${ }_{21}^{21}$ CABET, Etienne, Voyage en Icarie, Paris, Bureau du Populaire, 1848 [trad. it. Viaggio in Icaria, 1840, Napoli, Guida, 1983].

${ }^{22}$ BELLAMY, Edward, Looking Backward: 2000-1887, Boston, Ticknor and Co., 1888 [trad. it. Uno sguardo dal 2000, Soveria Mannelli, Rubbettino, 1991].
} 
opere letterarie inquadrabili nel genere utopico, perché questo permette di contrapporre un mondo antico, che deve essere criticato, a un altro, al quale si giunge attraverso un viaggio, indipendentemente dal fatto che sia effettuato attraverso uno spostamento nello spazio, nel tempo o nel mondo dei sogni. Richiamiamo questo aspetto solamente come un segnale della sostanza dell'intertestualità che percorre la storia di questo genere ${ }^{23}$.

Potremmo aggiungere ancora, come tratto caratteristico del genere utopico, la tendenza verso un determinato stile narrativo, nel quale la narrazione si subordina alla descrizione della città ideale, obiettivo principale di questi romanzi. Spesso questa città ideale si fonda su una "perfezione istituzionale" che viene minuziosamente descritta dall'autore ${ }^{24}$, il quale si impegna ad offrire, attraverso la sua opera, una schematizzazione della realtà letteraria da lui costruita. Per questa ragione il modello più consueto che soggiace al genere utopico, è quello delle topiche legate ai diversi problemi affrontati dal desiderio di realizzare e organizzare una società ideale che tessono il filo della narrazione, qui trasformato in un itinerario lungo il quale vengano passati in rassegna i problemi all'ordine del giorno ${ }^{25}$. Questo modello plasmato nel percorso didattico si unisce con un'altra caratteristica essenziale presente in questo modello narrativo: lo specchiamento - esplicito o implicito - dell'utopia nella civilizzazione che si intende criticare.

La strategia discorsiva e cognitiva nella maggior parte dei casi fa ricorso a quello che molti autori chiamano «straniamento» 0 «estrangement» ${ }^{26}$. A partire da queste promesse si può aggiungere che un'ulteriore caratteristica importante del genere utopico sia il fatto che l'utopia - o la descrizione

\footnotetext{
${ }^{23}$ Diversi autori sottolineano come il viaggio sia anch'esso strutturale per il genere utopico; altri evidenziano come il viaggio si dimostri una transizione imprescindibile, una sorta di iniziazione. MINERVA, Nadia, op. cit., p. 42; FORTUNATI, Vita, Scrittura di viaggio e scrittura utopica tra realtà e finzione, in BACCOLINI, Raffaella, FORTUNATI, Vita, MINERVA, Nadia (a cura di), Viaggi in Utopia, Ravenna, Longo Editore, 1996, pp. 13-19, p. 10.

${ }^{24}$ CIORANESCU, Alexandre, op. cit., p.53. Per una discussione sulla perfettibilità dell'utopia, si veda: RACAULT, Jean-Michel, op. cit., p. 32.

${ }^{25}$ Michel Racault, uno degli studiosi più autorevoli delle utopie, si spinge ad affermare come caratteristica del genere utopico l'intenzione di avvicinarsi all'idea per cui il narratore di un viaggio utopico elabori una specie di «ricostruzione antropologica totale», che comporta «istituzioni, una struttura sociale, un'economia, una religione, una lingua, etc, dal momento che ogni elemento è correlato a tutti gli altri». RACAULT, Jean-Michel, op. cit., p. 31. L'idea per cui sarebbe una caratteristica obbligata dall'utopia alla totalità, estensibile a tutti gli aspetti della vita umana, viene sostenuta anche da Luigi Firpo. FIRPO, Luigi, op. cit., p. 229.

${ }_{26}$ SUVIN, Darko «Um breve tratado sobre a Distopia 2001», cit., p. 468. «Straniamento [...] è una strategia percettiva in cui la valutazione è basata sul desiderio di critica radicale. Essa comporta molteplici possibilità di anamorfosi ed eversione degli aspetti salienti del mondo dell'autore e ha come proposito il riconoscimento del fatto che il lettore viva realmente un un mondo di valori sottosopra». SUVIN, Darko «Um breve tratado sobre a Distopia 2001», cit., pp. 468-469. Il concetto è stato sviluppato più dettagliatamente in Metamorphoses of Science Fiction: On the Poetics and History of a Literary Genre (SUVIN, Darko, Metamorphoses of Science Fiction: On the Poetics and History of a Literary Genre, New Haven, Yale University Press, 1979), tradotto in italiano com il titolo Le Metamorfosi della fantascienza (SUVIN, Darko, Le metamorfosi della fantascienza. Poetica e storia di un genere letterario, Bologna, Il Mulino, 1985). Una collettanea di saggi sul concetto è stata organizzata da Patrick Parrinder: PARRINDER, Patrick (ed.), Learning from Other Worlds: Estrangement, Cognition, and the Politics of Science Fiction and Utopia, Liverpool, Liverpool University Press, 2001. Si veda anche l'articolo: SUVIN, Darko, «Entrangement and Cognition», in Strange Horizons, 24 novembre 2014, URL: < http://www.strangehorizons.com/2014/20141124/1suvin-a.shtml > [consultato il 7 febbraio 2017].
} 
della stessa - in questo caso venga sempre impiegata come uno «strumento critico» ${ }^{27}$. Come conferma delle nostre considerazioni - e del fatto realmente notevole per cui l'insieme delle caratteristiche fin qui descritte sia perfettamente contemplato dalle diverse creazioni letterarie in sintonia con il genere utopico- possiamo affermare che esiste la possibilità effettiva di considerare queste creazioni letterarie, benché ciascuna sia dotata della sua storicità, di specificità e contingenze, nel novero di un genere comune.

Lasciamo per ultimo un tratto che appartiene tanto alle opere letterarie che costituiscono il genere utopico, quanto ai progetti e alle realizzazioni che ne costituiscono il pensiero e l'azione. Le utopie lato sensu si riferiscono sempre ad esperienze sociali. Conviene ricordare, se siamo nell'ambito del genere utopista, che si tratta di descrivere società; se siamo nel campo dei progetti o delle realizzazioni utopiste, si tratta invece di guardare alle esperienze sociali. La ricerca di un'avventura al di fuori del consorzio umano, come avviene nel caso delle opere letterarie ispirate dal modello di Robinson Crusoe ${ }^{28}$, non costituisce dunque un'utopia, così come non la costituisce il modello concreto dell'eremita che si autoesilia.

Desideriamo ancora affermare che, benché non esista una necessaria continuità tra le varie realizzazioni oggi definite utopiche e che ne troviamo qui un'infinità di casi differenti, l'esercizio intellettuale di far coincidere problemi specifici di queste opere letterarie e delle elaborazioni utopiste del mondo sociopolitico può contribuire alla comprensione di aspetti molto interessanti del pensiero sociale, particolarmente degni di riflessione e studio. La riflessione a cui desideriamo dedicarci ora verte sul problema specifico dell'uguaglianza e, più specificamente, di come viene trattata da Cabet, facendo ricorso all'intertestualità con altre opere utopiche. È opportuno anche affermare chiaramente che ci soffermeremo fondamentalmente sulla costruzione letteraria realizzata da Cabet nel suo romanzo Voyage en Icárie, e non tanto sui successivi tentativi di questo autore e attivista politico volti a realizzare un'esperienza socialista comunitaria, sebbene in qualche occasione, in questo articolo, potremo menzionarle ${ }^{29}$. L'oggetto della nostra analisi sarà infatti l'Icarie immaginata da Cabet - che, così come molte costruzioni utopiche, vive in una realtà parallela,

\footnotetext{
${ }^{27}$ PRÉVOST, André, «A utopia: o gênero literário», in Morus. Utopia e Renascimento, 10, 2015, pp. 437-448, p. 446 [art. orig.: «L'utopie: le genre litteraire», in Moreana, 8, 31-32, 3/1971, pp. 161-168.

${ }^{28}$ DEFOE, Daniel, Robinson Crusoe, London, W. Taylor, 1719.

${ }^{29}$ Gli sforzi concreti di Cabet per fondare e sviluppare una comunità icariana - che risultò ben diversa dalla grande città da lui idealizzata nella sua costruzione letteraria Voyage en Icarie - sono stati studiati da diversi autori diversi: SHAW, Albert, Icaria: A Chapter in the History of Communism, London, Kessinger Publishing, 2010 [ed. orig., New York-London, G.P. Putnam's Sons, 1884]; SUTTON, Robert P., Les Icariens: The Utopian Dream in Europe and America, Urbana, University of Illinois Press, 1994; LARSEN, Dale R (ed.), Soldiers of Humanity: a History and Census of the Icarian Communities, Nauvoo (Il), The National Icarian Heritage Society, 1998; WIEGENSTEIN, Steve, «The Icarians ant their Neighbors», in International Journal of Historical Archaeology, 10, 3/2006, pp. 289-295. Le fonti per questo studio possono essere trovate nella documentazione pubblicata dallo stesso Étienne Cabet: CABET, Étienne. Colonie Icarienne aux États-Unis D’Amérique: sa constitution, ses lois, as situation matérielle et morale après le premier semestre 1855, New York, Lenox Hill Pub.\& Dist. Co., 1856.
} 
«sovrapposta alla nostra» ${ }^{30}$ - e non l'esperienza icariana che successivamente catalizzò le attenzioni di Étienne Cabet con la fondazione di una colonia negli Stati Uniti d'America.

Prima di giungere a questa analisi più generale della Icarie letteraria con l'intento di preparare meglio il terreno al problema specifico che esamineremo nella società immaginata da Cabet, sarà opportuno mobilitare un sistema concettuale che permetta di stabilire un legame comparativo fra le analisi che verranno portate avanti. Partiremo da una riflessione sui diversi ambiti attraverso i quali si possono distinguere i differenti tipi di uguaglianza.

\section{Due domande fondamentali sull'uguaglianza}

Un buon riferimento metodologico per la comprensione dei sistemi che discutono l'uguaglianza è stato proposto da Norberto Bobbio nella sua Teoria generale della politica. Per il politologo italiano ogni sforzo nell'intento di riflettere sistematicamente su questo tema deve partire da due domande fondamentali. Quando si affronta il tema dell'uguaglianza, una prima domanda è: «uguaglianza tra chi?». In concomitanza, ci si domanda subito: « uguaglianza di che cosa?» ${ }^{31}$. Inoltre bisogna considerare che questa seconda domanda, "uguaglianza di che cosa», può implicare vari sviluppi che, per utilizzare un'espressione di Amartya Sen $^{32}$, potremmo qui chiamare di «variabili focali». Ogni variabile focale definisce uno spazio al quale si riferisce l' uguaglianza proposta o pretesa. Può essere proposta l' uguaglianza di qualcosa come la scelta dei rappresentanti, le opportunità di lavoro, di istruzione e, al limite, l' uguaglianza di tutto. Successivamente si deve considerare che le domande iniziali unite («uguaglianza tra chi» e « uguaglianza di che cosa») si possono ramificare in quattro possibili risposte che, secondo Bobbio, sarebbero le seguenti: uguaglianza di alcuni in relazione a qualcosa; uguaglianza di alcuni in tutto; uguaglianza di tutti in qualcosa; uguaglianza di tutti in tutto. È possibile schematizzare questo piano di categorie secondo il quadro che presentiamo di seguito:

\footnotetext{
${ }^{30}$ «Gli abitanti delle Città Felici non vivono neppure una storia parallela alla nostra; abbiamo già detto in precedenza che si trovano in un tempo che si sovrappone al nostro». BACZKO, Bronislaw, «Utopia», cit., pp. 356-357.

${ }^{31}$ BOBBIO, Norberto, A teoria das formas de governo, Brasília, UnB, 1997 [ed. orig.: La teoria delle forme di governo nella storia del pensiero politico, Roma, Giappichelli, 1975].

${ }^{32}$ SEN, Amartya K., Inequality Reexamined, Cambridge, Harvard University Press, 1992, passim.
} 


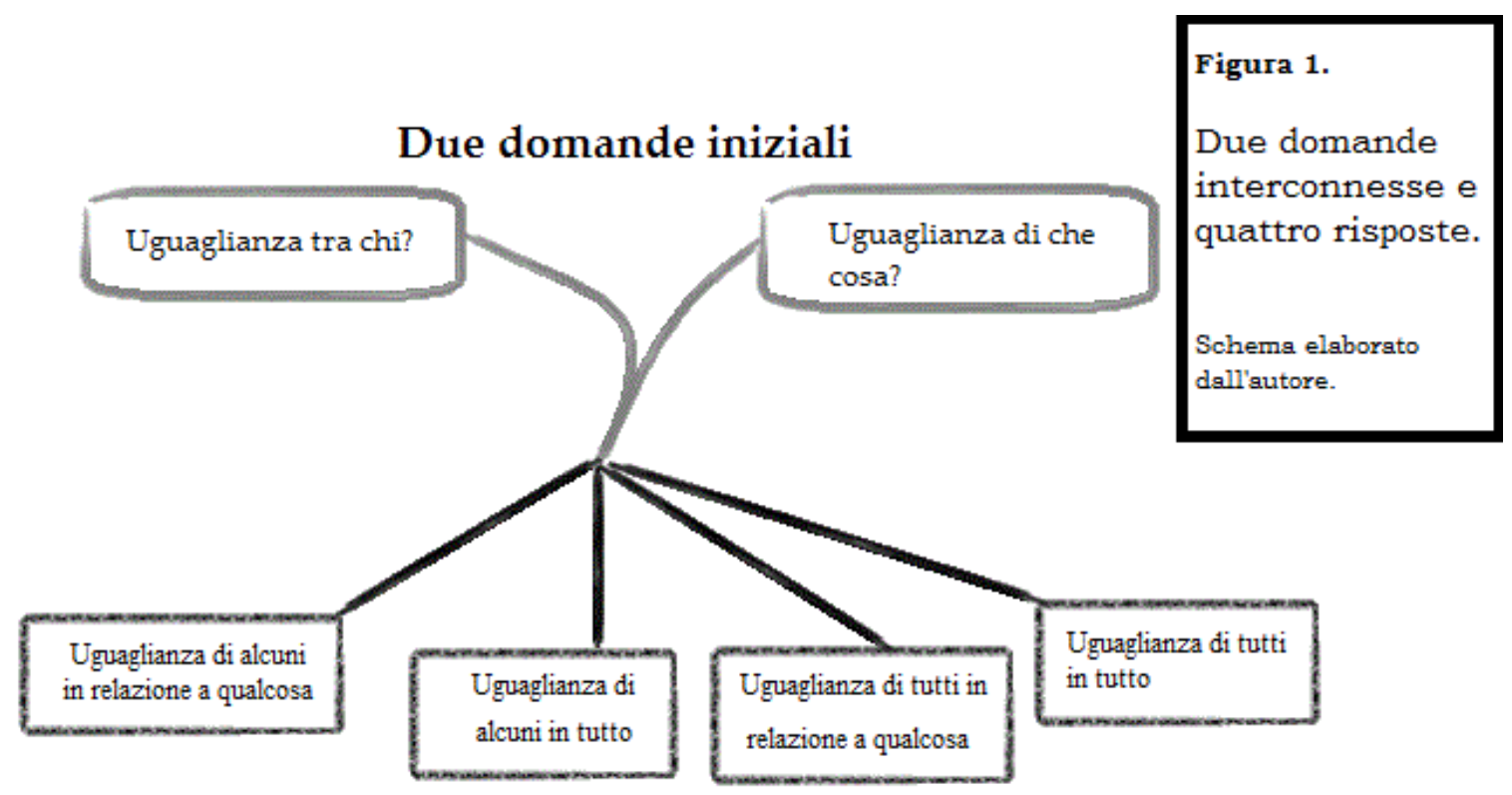

\section{Quattro risposte possibili}

Di esempi inquadrabili in ciascuno di questi casi se ne possono trovare a profusione nella storia delle idee e nella storia. Non vale molto la pena insistere sulla prima possibilità (uguaglianza di alcuni in relazione a qualcosa). Come sottolineato da Bobbio, questo è il luogo comune di qualsiasi legge o regolamento. Ciascuna legge si riferisce a soggetti che hanno qualcosa in comune in grado di inquadrarli nella situazione specifica di cui tratta la legge - e che in questo modo corrispondono solamente a un sottoinsieme del macrocosmo dei cittadini. Al contempo la legge stabilisce diritti o pene in merito a qualche aspetto. Riassumendo la questione, una legge si riferisce a individui specifici in relazione a un problema ugualmente specifico.

La risposta numero 2, così come le altre, ci conduce verso l'avventura umana del pensiero politico. «Uguaglianza di alcuni in tutto» era ciò che propugnava Platone nella Repubblica. Platone era critico nei confronti del modello politico della sua stessa polis - l'Atene classica che si sarebbe trasformata in una democrazia piena, includendo ampi diritti politici per una parte ridotta della popolazione (pur conservando l'esclusone degli schiavi, degli stranieri e, su un altro piano, delle donne). Tra tanti utopisti del XIX secolo vedremo emergere la proposta numero 4: «uguaglianza di tutti in tutto». Come rendere possibile un tale sogno di egualitarismo? Per procedere sarà utile riflettere sulla distinzione che si può operare tra modelli egualitari e modelli egualitaristi.

\section{Egualitarismi}

All'interno dello schema proposto in precedenza , la risposta numero 4 («uguaglianza di tutti in tutto») ci riporta alle utopie e alle proposte egualitariste. Non ci sarà modo di esaminare tutti gli 
immaginari egualitaristi nei limiti imposti da questo articolo, ma si può dire di sfuggita che le due risposte immediatamente precedenti ( numeri 2 e 3 ) corrispondono, ciascuna a modo proprio, a principi egualitari di qualche tipo. La Repubblica di Platone - del secondo tipo un - è un'utopia egualitaria all'interno dei limiti che si propone: prevede l'esclusione di quelli che sono fuori da questi limiti e contempla livelli sociali e funzioni specifiche. Si può dire anche che lo sia persino il modello proposto da John Locke e da altri autori del XVII e XVIII secolo, che dimostrano come una prospettiva liberale circoli anche all'interno di una proposta egualitaria ma non egualitarista («uguaglianza di tutti in alcuni aspetti»). Chi negherà che la Dichiarazione dei diritti dell'uomo sia uno splendido documento egualitario negli articoli che descrivono i diritti inalienabili degli esseri umani? E ovunque le democrazie liberali si rafforzano attraverso l'immaginario dell'uguaglianza, benché la loro base capitalistica si sostenti ampiamente grazie alla disuguaglianza.

Questi due modelli - repubblica platonica e democrazia liberale - si sviluppano all'interno di limiti egualitari. Ma l'ambito entro cui si sviluppano diverse delle utopie ottocentesche - tra le quali la società creata letterariamente da Cabet - si riferisce all'egualitarismo. C'è una sottile distinzione sarà opportuno sottolinearlo sin d'ora - tra egualitario ed egualitarista. Se osserviamo bene, esiste un superlativo sufficientemente significativo che si sviluppa nel passaggio da una parola all'altra. L'egualitarismo propone la pienezza dell'uguaglianza nei due termini dell'equazione: «Uguaglianza per tutti in tutto (o quasi tutto)»: soggetto e oggetto del processo di equalizzazione vengono estesi tanto quanto è possibile. Ad uno degli estremi, il soggetto dell'uguaglianza tende all'unità assoluta e spariscono le classi sociali; all'altro, l'oggetto dell'uguaglianza si estende a tutte le condizioni di vita e, sintomaticamente, può scomparire la proprietà privata. Non è necessario che vengano raggiunti questi limiti perché già si possa parlare di egualitarismo, ma in qualche maniera dovremo rimanere più vicini proprio a questi poli.

Per comprendere i differenti modelli e le matrici egualitariste, possiamo riprendere per sommi capi la loro storia. Ad eccezione delle esperienze più limitate del comunismo cristiano primitivo, le utopie e i modelli politici egualitaristi iniziano a consolidarsi nella modernità. Un punto di riferimento teorico particolarmente importante è sicuramente la Cospirazione degli uguali di Babeuf, testo scritto da Filippo Buonarroti nel $1828^{33}$. Del resto le utopie egualitarie ed egualitariste già avevano iniziato ad apparire nella letteratura - come abbiamo già visto - sin dall'umanesimo rinascimentale, in particolar modo con l'Utopia di Moro (1478-1535) e la Città del Sole di Campanella (1568-1639), due riferimenti importanti per il genere utopico. Assoceremo a una matrice comune, che denomineremo "matrice Moro", queste utopie egualitarie che prevedono un mondo abitato da esseri saggi o speciali - e che, pertanto, sono pronti per una vita tanto esente da tensioni quanto è possibile che lo sia (una vita che, per la verità, non può esistere effettivamente se non fuori da

\footnotetext{
${ }^{33}$ BUONARROTI, Philippe, Babeuf's conspiracy for equality, London, Hetherington, 1836.
} 
questo mondo). È così che l'Utopia di Moro, descritta nell'opera omonima che venne pubblicata nel 1516, si presenta come una società immaginaria e idealizzata nella quale non esiste la proprietà privata e si verifica una assoluta comunione dei beni e della terra, in assenza di antagonismi tra la città e la campagna - tratto utopico attraverso cui l'autore indirizza una critica all'Inghilterra della sua epoca. Allo stesso modo, non esiste lavoro salariato, né esistono spese superflue o lussi eccessivi e necessari, dal momento che lo Stato ricopre il ruolo di organo amministratore della produzione. Storicamente localizzata in un contesto ben definito, quest'opera dell'immaginazione utopica rinascimentale costituisce simultaneamente una critica alla società feudale e all'Inghilterra contemporanea a Moro. Conserva il valore pionieristico, tra le creazioni letterarie che si inquadrano nel genere utopico, di essere stata la prima idealizzazione di una società in comunione dei beni.

L'organizzazione politica è democratica, grazie all'elezione diretta dei magistrati e della suprema autorità del principe. Ma è interessante osservare il sistema fortemente autoritario previsto per evitare che vi siano cospirazioni, trame e manipolazioni dell'opinione pubblica. Qualsiasi riunione politica al di fuori del Senato e delle Assemblee Popolari, dove e quando sia prevista la discussione e la votazione periodica delle leggi, viene punita con la morte. Esistono istituzioni permanenti la cui funzione è impedire che i governanti cospirino contro la libertà o esercitino qualche forma di tirannia. Del resto, a parte questo regime autoritario d'eccezione escogitato per far fronte alle situazioni che minaccino il funzionamento democratico, l'Utopia è il luogo privilegiato della libertà di pensiero. Moro costruisce un vero paradiso di tolleranza religiosa, dal momento che gli abitati di Utopia professano i credi più vari senza che questi entrino in contrasto fra loro.

La matrice egualitaria presentata dall'autore presuppone l'esistenza di uomini savi, pronti per un mondo di libertà ed eguaglianza, senza ansie egoiste di qualsiasi sorta. Un mondo di uomini che non esistono, potremmo aggiungere. Mondo, oltretutto, senza storia poiché egli non spiega come sia stato possibile giungere a questo stato di perfezione politica a partire da un mondo in precedenza abitato da esseri comuni che, benché si stessero preparando per l'uguaglianza, rimasero senz'altro coinvolti da tensioni sociali di tutti i tipi, coltivando gli interessi egoistici che sorgono spontaneamente in una società competitiva. Rinunciando a esaminare il passaggio dal mondo imperfetto al mondo utopico, Moro tralascia anche la questione della presentazione di un programma rivoluzionario o di trasformazione sociale. Condanna il suo creato perfetto a uno spazio immaginario, a un "non-luogo" proibito agli uomini che rimangono invischiati nella storia e che hanno bisogno di costruire la società in cui andranno a vivere con i loro brancolamenti ed esitazioni, le loro imperfezioni e rivoluzioni.

Altre proposte utopiche che si avvicinano al modello presentato da Moro cominciano ad affiorare con l'umanesimo rinascimentale: successivamente viene pubblicata la Città del sole di Campanella (1568-1639) e la New Atlantis di Bacon (1561-1626). Queste proposte utopiche si poggiano o sull'idea che quelli che le abitano sono pronti per la felicità, per l'uguaglianza e per la libertà - discostandosi 
un po' dai conflitti occasionali e di interessi che possono verificarsi nelle esperienze reali - oppure sull'idea di un'élite di saggi rispettati incondizionatamente da tutti e che per proprio per questo riescono a risolvere qualsiasi problema sociale scegliendo infallibilmente la miglior soluzione. Con quest'ultima variante entriamo nel campo di una piccola élite di governanti perfetti, che con la loro presenza e direzione "utopizzano" l'ambiente . Questa distanza tra una piccola cerchia di governanti e la società in favore della quale essi agiscono, con autoritarismo ma anche con il sacrificio personale, è una caratteristica di questi modelli utopici, che li rende distinguibili da quello di cui discuteremo successivamente (l'utopia di Edward Bellamy).

La Città del Sole (1602), di Campanella, ad esempio, oltre ad ispirarsi all'Utopia di Moro, il suo modello immediato, si mostra sufficientemente temperata con elementi originari della Repubblica di Platone. Ciò che fa della Città del Sole un'utopia egualitarista è, sopra ogni cosa, il fatto che i beni siano messi in comune. Non esiste la proprietà e viene impartita un'educazione egualitaria. L'egualitarismo traspare dagli stessi indumenti, poiché tutti vestono abiti uguali. L'egualitarismo viene meno quando entra in scena la questione dei generi maschile e femminile; di fatto, i due sessi sono trattati in modo differente - se non con disparità, quantomeno con differenze sufficientemente chiare, che iniziano già a un certo stadio dell'educazione impartita ai bambini. D'altra parte, anche tratteggiando un completo egualitarismo, la Città del Sole prevede criteri di distribuzione basati sul merito.

Il dettaglio che ci interessa è che questa utopia si rafforzi anche nella direzione e nel governo imposti da un gruppo selezionato di saggi - un gran consiglio di magistrati organizzati con uno schema piramidale, che ha al suo vertice quattro saggi maggiori, uno dei quali ricopre il ruolo di autorità massima. L'Hoh, parola che significherebbe "il Metafisico", è il sommo saggio che si trova al vertice del consiglio . A lui sono legati gli altri tre saggi: "Potenza", "Amore” e "Sapienza" - ciascuno dei quali riunisce settori importanti della vita sociale e dei campi umani del pensiero e dell'azione. Così "Potenza" è il responsabile finale per tutto ciò che è relativo alla pace e alla guerra; "Amore" sovraintende a tutto ciò che è in rapporto con l'alimentazione, il vestiario e l'unione amorosa; infine "Sapienza" regge le arti, le scienze e l'istruzione. La piramide della saggezza si estende molto oltre. A ciascuno di questi tre saggi si lega un certo numero di magistrati. Per esempio, il ministro "Sapienza" sovraintende un circolo ristretto di undici magistrati (Astrologo, Cosmografo, Geometra, Storico, Poeta, Logico, Retorico, Grammatico, Medico, Fisiologo, Moralista). In questo modo viene costituita la piramide dei sapienti che, separatamente, sovraintende alla vita degli Uguali che abitano la Città del Sole. Quantunque i saggi facciano in ultima istanza parte dal corpo stesso dei cittadini, non viene meno quella società egualitaria che necessita, per poter essere diretta efficacemente, di un corpo a parte - una sorta di organo speciale all'interno del proprio corpo - che presti attenzione affinché tutto vada nella direzione della soddisfazione e perché l'organismo sociale si sviluppi. 
Un altro esempio di genere utopico rinascimentale è la New Atlantis di Bacon, opera pubblicata postuma nel 1627. L'Atlantide immaginaria proposta da Bacon si oppone a quella che Platone aveva descritto nella Repubblica. La comunità utopica di Bacon vede anch'essa il suo benessere assicurato da un gruppo di saggi che abita la Casa di Salomone: un'istituzione scientifica la cui funzione è ricercare continuamente i mezzi per semplificare la vita e di dirigere la vita dei cittadini con l'intento di assicurare loro la felicità. Ma è in particolare un gruppo di scienziati a lavorare incessantemente per l'ampliamento della conoscenza in tutti i campi. Questa istituzione è il vero cuore dell'utopia proposta da Bacon, dal momento che si lega ai centri agricoli, a quelli sanitari, a quelli deputati alla produzione dell'energia e così via. I saggi sono - in questo caso - lavoratori instancabili che offrono il loro talento scientifico per il beneficio dell'intera comunità, a cui non rimane che usufruire di un mondo di libertà e uguaglianza perfettamente armonizzato da scienza e sapienza. Un dettaglio rilevante è che la New Atlantis di Bacon già possiede una storia. L'Utopia di Tommaso Moro e la Città del Sole di Campanella non solo sono presentate come "non-luoghi" (utopie), ma anche come realtà fuori del tempo - benché non si spieghi come si sia giunti fino ad esse. Nella New Atlantis di Bacon rinveniamo un'origine: questa è stata fondata 900 anni prima del racconto da un grande saggio altruista proveniente dall'esterno. L'utopia baconiana, in questo modo, viene posta in essere attraverso un gesto magnanimo - non è generata da un processo rivoluzionario o da riforme graduali.

Nel XIX secolo nacquero proposte utopiche che, in qualche modo, costituiscono variazioni più moderne dell'utopia di Moro. Nacquero pure i "progetti utopisti", anche se bisogna notare che, in questo caso, le proposte utopiche erano rafforzate dall'intenzione di vedersi un giorno concretizzate, sebbene, in definitiva, non furono coronate da successo. In comune con il modello egualitarista precedente avevano una rigorosa pianificazione imposta dall'alto. Il primo riferimento sono le idee di Charles Fourier (1772-1837), che tramutò in realtà un "progetto utopista" (e non un'opera letteraria apparentabile al genere utopico). Scrivendo di un mondo più avanzato in termini industriali, e che ha assistito ai regressi della Rivoluzione francese, Fourier pretende di portare avanti il suo sogno di una società giusta ed egualitarista con la compiacenza di "capitalisti illuminati" in sintonia con le idee liberali. Il grande fondatore, d'altro canto, sarebbe stato egli stesso; dunque non un fondatore immaginario, ma storicizzato, che tuttavia non riuscì a realizzare il proprio progetto.

Le falangi proposte da Fourier corrispondevano a piccole unità sociali con una popolazione di circa 1500 abitanti e ciascuna disponeva di un edificio comune chiamato falansterio, dove tutti vivevano armonicamente ${ }^{34}$. A prescindere da un calcolo relativo all'abbinamento numerico di

\footnotetext{
${ }^{34}$ In un'opera chiamata La Teoria dei quattro movimenti (1808), Fourier era giunto alla curiosa conclusione per cui il falansterio avrebbe dovuto ospitare una popolazione di 1620 persone (810 di ciascun sesso). Fourier giunse al numero di 1620 a partire dalla teoria - che avrebbe poi sviluppato - dei Quattro Movimenti, secondo
} 
uomini e donne, l'idea era quella della spontanea dissoluzione nel falansterio di formazioni sociali rudimentali come la cellula familiare monogamica e ristretta. Per questa ragione esso prevedeva molti spazi di socializzazione, inclusi i refettori comuni. Fourier era un critico feroce del matrimonio, e riteneva che eliminandolo tutto il resto sarebbe venuto di conseguenza, in modo naturale, portando ad una società realmente libera. La rottura naturale con la monogamia era un elemento previsto nello sviluppo dei falansteri. L'attitudine alla moltiplicazione dei rapporti amorosi era definita da Fourier "angelica". Egli inneggiava a un comportamento edonista nel quale tutti avrebbero cercato il massimo del piacere (quel che già abbiamo visto nell'Utopia di Moro). Il denaro e la proprietà privata, ad esempio, non sarebbero stati soppressi nonostante nella vita "societaria" e salutare del falansterio, epurata dai modelli dell'egoismo e dell'individualismo che fino ad allora avevano caratterizzato la cosiddetta società "civilizzata", né il denaro, né la proprietà avrebbero avuto un qualsiasi effetto dannoso e generatore di oppressione economica.

Per quel che riguarda il modello di redistribuzione della ricchezza, si prevedeva che questo sarebbe stato orientato in funzione della qualità del lavoro prodotto da ciascuno, il che avrebbe giustificato le differenze secondo giustizia. Beninteso: non sarebbe stata una remunerazione che avrebbe privilegiato propriamente tipologie differenti di lavoro, ma semplicemente l'efficienza e il beneficio reale che i gruppi professionali stavano producendo per la comunità. Quanti meno infermi avesse avuto un falansterio, tanto più i medici che operavano al suo interno sarebbero stati ben remunerati; quanti meno problemi strutturali o di manutenzione avessero colpito l'edificio, tanto più avrebbero guadagnato gli ingegneri e i tecnici; quanto più deliziosa e nutritiva fosse stata l'alimentazione, tanto più avrebbero guadagnato i cuochi o coloro i quali in quel mese si fossero dedicati alle attività di sostentamento. In questo modo ciascun gruppo funzionale avrebbe fatto uno sforzo collettivo per innalzare lo standard dei risultati del suo lavoro e, in questo modo, i propri guadagni.

Anche l'industriale Robert Owen (1771-1858), un uomo di azione che contribuì significativamente alla storia dei progetti utopisti, tentò a d'istituire una società riformata, investendo le sue risorse in colonie cooperative nelle quali la proprietà privata sarebbe stata eliminata. Egli già aveva ottenuto qualche successo nella sua attività industriale organizzando le sue fabbriche in modo sorprendentemente umano e con grande giustizia sociale, senza che questo pregiudicasse lo sviluppo capitalista delle imprese. Tuttavia, i tentativi di concretizzazione del suo progetto realmente egualitarista naufragarono, in parte a causa delle molte resistenze da parte dell'aristocrazia inglese del periodo ${ }^{35}$.

cui gli esseri umani sarebbero stati mossi da tredici passioni specifiche. La popolazione iniziale del falansterio doveva essere calcolata in modo che tutti nella comunità avessero l'opportunità di incontrare altri con caratteristiche complementari alle proprie.

${ }^{35}$ Le idee di Owen vennero raccolte in una collettanea di saggi, pubblicata nel 1820. OWEN, Robert, A new view 
Infine, quale ultimo esempio per illustrare il gruppo degli ideatori di "progetti utopisti", troviamo una società governata da un consiglio di saggi e artisti proposta dal conte Claude-Henri Saint-Simon (1760-1825). Aziché rigettare interamente il mondo, l'utopia di Saint-Simon prevedeva una società industriale privata di disordini e ingiustizie, dal momento che sarebbe stata condotta consensualmente dai "produttori": gli operai, gli imprenditori, i saggi, gli artisti e i banchieri. In una delle sue prime opere, Lettres d'un habitant de Genève à ses contemporains (1803), egli già aveva proposto che gli scienziati prendessero il posto delle autorità religiose nella direzione spirituale delle scienze moderne. Tuttavia, nell'anno della sua morte, avrebbe pubblicato un'opera intitolata Nouveau christianisme (1825), che si occupava dell'idea di una riforma in campo religioso ${ }^{36}$. Giova anche ricordare che, come Fourier, Saint-Simon ebbe una preoccupazione peculiare nel discutere la necessità di correggere le diseguaglianze di genere: possiamo notare come tra i sansimoniani si trovino alcune delle donne pioniere nella lotta per i diritti femminili e per un loro inserimento basato sull'eguaglianza nel mondo sociale e politico. Tra queste si deve ricordare il nome di Claire Bazard (1794-1883). Oltre a ciò, relativamente alle modalità di distribuzione della ricchezza, SaintSimon preconizzava - in Du système industriel (1822) - la formula «a ciascuno secondo le proprie capacità, a ciascuna capacità secondo il proprio lavoro» ${ }^{37}$.

\section{L'Icarie di Étienne Cabet}

L'ambiguità dei progetti utopisti che imperversarono prima e durante il XIX secolo, come sarebbe successivamente stato messo in rilievo dalla forte critica nei confronti dei socialismi utopici portata avanti da Marx ed Engels, si deve in parte alla credenza secondo cui la realizzazione delle loro proposte socialiste sarebbe stata possibile solamente attraverso l'azione (in genere autoritaria) di un unico leader o di un gruppo di saggi. La figura dell'unità dirigente che veglia sulla società per assicurare il buon funzionamento dell'ordine era abbastanza comune nelle utopie letterarie - quelle che davano vita ad una società immaginaria attraverso una trama di finzione inquadrabile nel "genere utopico", sull'esempio delle città idealizzate da Moro, Campanella o Bacon - come nelle

of society and other essays, London, Penguin Books, 1991.

${ }^{36} \mathrm{Su}$ questo tema, cfr. DESROCHE, Henri, Saint-Simon. Le nouveau christianisme et les Écrits sur la religion, Paris, Seuil, 1969. Sul pensiero utopico di Saint-Simon, si veda: PETRE-GRENOUILLEAU, Olivier, Saint-Simon, l'utopie ou la raison en actes, Paris, Payot, 2001; MUSSO, Pierre. Saint Simon et les saint-simonisme, Paris, PUF, 1999.

${ }^{37}$ Saint-Simon fu, tra i socialisti utopici, uno di quelli che in misura maggiore fecero proseliti nel XIX secolo (i sansimoniani) oltre ad annoverare fra i suoi ammiratori scrittori romantici come Victor Hugo e George Sand. Tra i sansimoniani propriamente detti - quelli che diedero continuità al movimento e pubblicarono Doctrine de Saint-Simon: exposition - i nomi di rilievo sono quelli di Saint-Amand Bazard (1791-1832) e Barthélemy Prosper Enfantin (1796-1864). Entrambi, dopo una fase iniziale di pratiche rivoluzionarie tra i carbonari francesi, finirono per aderire, rispettivamamente nel 1828 e nel 1825, al sansimonismo. 
realizzazioni utopiste che si presentavano sotto forma di progetti da realizzare effettivamente in un preciso momento, come le proposte di Fourier, Saint-Simon e Owen.

Nella realtà letterariatutto pare funzionare come un orologio. Seguendo l'immaginazione dell'autore, i conflitti sembrano non esistere e, quando esistono, le strade per la loro risoluzione sono già tracciate. Allo stesso modo, tanto i leader utopici quanto la popolazione vengono qui idealizzati. Possiamo fare supposizioni, tuttavia, su ciò che sarebbe accaduto nel passaggio da un'utopia letteraria a un'esperienza reale. Che cosa sarebbe avvenuto se esseri umani in carne e ossa avessero tentato di mettere in pratica una Città del Sole, una Nuova Atlantide, un'Utopia? Fourier desiderava effettivamente costruire i suoi falansteri - da lui idealizzati in diverse opere - ma il suo progetto non si scostò mai dalle pagine e dal sogno. Sarebbe stato deluso dalla realtà effettiva umana e troppo umana - se ci fosse stata un'opportunità concreta di realizzare una nuova società che sognava sotto forma di grandi falansteri? Come avviare - se desideriamo porre tutto in termini più semplici - il passaggio dall'utopia alla topia, dal non luogo al luogo?

L'utopia letteraria ottocentesca che esamineremo maggiormente in profondità, considerando anche la sua inusitata ricezione da parte di una moltitudine di lettori che tentarono di realizzarla fattivamente, ci pone di fronte ai dilemmi e alle ansie generati dall'ambizione di trasformare un'utopia letteraria con i sogni e le parole in una realtà tangibile, fatta di carne, ossa e pietra. L'autore di questa nuova esperienza fu il francese Cabet (1788-1856), avvocato e politico attivo durante la Restaurazione e sotto il governo monarchico di Luigi Filippo. Egli si impegnò da subito nella pratica rivoluzionaria avendo preso parte alla società segreta dei carbonari. Occupò una carica importante nel governo orleanista, portato al potere dalla rivoluzione borghese del 1830. Malgrado ciò, mantenne salda la sua posizione favorevole alla rivoluzione ed egli venne destituito dal suo incarico come rappresaglia per la sua pubblica presa di posizione in favore dei popolani ribelli nel $1831^{38}$. Una situazione simile, nel 1834, quando era deputato e editore del giornale «Le Populaire», lo portò infine ad essere esiliato in Inghilterra ${ }^{39}$.

$\mathrm{Fu}$ qui che ebbe inizio la carriera letteraria di Cabet. Influenzato dalla figura di Robert Owen, industriale utopico di cui abbiamo detto in precedenza, Cabet trasse ispirazione da lui per scrivere nel 1840 un'opera di grande successo: un'utopia letteraria intitolata Voyage en Icarie. In essa, Lord William Carisdall, il personaggio centrale della storia, visita un paese fantastico chiamato Icaria. Questa società ha raggiunto un'organizzazione in cui le disuguaglianze sono scomparse attraverso diversi processi- sempre assicurati da uno Stato rafforzato - come l'abolizione dell'eredità,

\footnotetext{
${ }^{38}$ La critica della dimensione conservatrice del governo monarchico di Luigi Filippo compare anche nella História da Revolução de 1830, di Cabet.

${ }^{39}$ Esistono eccellenti biografie sobre su Cabet, tra le quali quella di François Fourn, pubblicata recentemente. Lo spazio di riflessione di questo articolo, tuttavia, non ci permetterà di approfondire gli aspetti biografici. Sull'esperienza concreta di Cabet e degli icariani, un riferimento importante è l'opera Utopian Comunism in France: Cabet and the Icarians. JOHNSON, Christopher H., Utopian Comunism in France: Cabet and the Icarians, Ithaca (NY), Cornell University Press, 1974.
} 
un'alimentazione per tutti commisurata alle proprie necessità, la proprietà comune dei mezzi di produzione, un'organizzazione del lavoro dinamica all'interno di fabbriche nazionali e un efficiente sistema di pubblica istruzione. Icaria presenta anche elementi già riscontrati in altre utopie rette da uomini saggi, come la supervisione familiare e il controllo eugenico dei matrimoni. Una simile società perfetta dal punto di vista egualitarista sarebbe stata fondata da Icaro, dittatore benigno che, nei primordi della storia di questo paese immaginario situato in qualche luogo dell'Atlantico, avrebbe instaurato e governato il paese con saggezza ${ }^{40}$. In questo caso, possiamo dire che il modello utopico commisurato ad un'unità direttiva di saggi si riduceva, all'atto iniziale, a un unico uomo. Successivamente, si era stabilita una giunta, ossia un'interconnessione di comitati composti dagli individui in grado di deliberare nelle differenti aree delle necessità umane.

Il romanzo produsse un tale impatto sulla società francese, che un grande gruppo di ammiratori di Cabet manifestò il desiderio di realizzare, nella pratica, l'“icarianesimo". In un manifesto intitolato Allons en Icarie, Cabet lanciò l'appello perché un'“Icaria" venisse creata in Texas, negli Stati Uniti, una suggerimento di Owen. Nel marzo del 1848, i primi icariani francesi raggiunsero l'America, per prendere possesso di una terra che era molto lontana da quella che immaginavano quando avevano firmato un accordo con una compagnia americana. Del milione di acri di terra promessi alla firma del contratto, essi ne ebbero a disposizione solamente diecimila che allora non erano promettenti quelli promessi. Anche così Cabet portò avanti il suo piano per realizzare la sua utopia socialista, agricola e artigianale nello Stato dell'lllinois. Abbiamo detto in precedenza che avremmo parlato di un tentativo effettivo di trasformare un'immaginazione utopica in atto pratico o di trasporre il modello di un'utopia letteraria in una comunità reale. Così non fu per più di un motivo. Il principale di questi è che Icaria - la società immaginaria creata dalla finzione letteraria di Cabet - era uno Stato dall'estensione insulare considerevole, diviso in cento province di dimensioni simili, ciascuna di esse divisa in cento comuni con la propria capitale situata geometricamente al centro della regione. Diversamente da questo imponente paese immaginario, strutturato su di una spazialità quasi matematica, l'Icaria reale (o il tentativo di reificarla in una piccola regione di coloni negli Stati Uniti d'America) non sarebbe stato nulla più che un piccolo ammasso di capanne perso nella prateria statunitense, distante dai centri urbani e abitato da pionieri comunisti mossi dall'immagine letteraria di Cabet e innamorati del suo progetto sociale e politico. Concentriamoci, comunque, sull'Icaria immaginaria di Cabet. Il focus dello studio, in questo caso, non sarà sull'esperienza utopista di Cabet come fondatore di una colonia socialista, ma sulla sua inventiva letteraria nel produrre un'opera di impatto facente parte del "genere utopico".

\footnotetext{
${ }^{40}$ Il personaggio fondatore, Icaro, sarebbe una sorta di Napoleone idealizzato e perfetto. Lewis Mumford, che commenta la Icarie di Étienne Cabet nella sua opera The Story of Utopias, sottolinea questo aspetto: «Cabet visse i suoi anni più sensibili nel fulgore delle campagne napoleoniche e alla luce crepuscolare della tradizione napoleonica che prevalse anche dopo che le conquiste del generale erano sparite dall'orizzonte». MUMFORD, Lewis, História das Utopias, Lisboa, Antígona, 2007, p. 127.
} 


\section{Icaria: uguaglianza geometrica e uguaglianza sociale}

La capitale omonima del paese di Icaria è di analoga eleganza geometrica. È divisa in 60 municipi, ciascuno autosufficiente per ciò che concerne alcuni aspetti essenziali: spazi pubblici ben definiti, un'unità educativa, un ospedale, un tempio, differenti tipi di negozi. Queste scacchiera di comuni è tagliata a metà da un fiume che divide la città in due parti - con ogni probabilità questo è un richiamo alla Senna di Parigi nell'immaginazione cartografica di Cabet - la città è suddivisa da una trama rettilinea di 100 lunghe strade che si intersecano perpendicolarmente.

La forma esteriore della capitale è circolare. Ciò che più ci interessa, tuttavia, è l'organizzazione sociale e politica, la modalità con cui si stabilisce una società egualitaria a partire da questa spazialità geometrica, il modo in cui si organizza la vita umana nei suoi diversi campi di attività. La disponibilità e l'equilibrio dell'alimentazione vengono stabiliti da una commissione di scienziati in grado di deliberare con cognizione di causa sul tema nutrizionale. Il lavoro, sotto l'egida dello Stato, è organizzato all'interno di fabbriche nazionali e il suo prodotto viene depositato in magazzini pubblici. A provvedere alle abitazioni, al vestiario, ai trasporti, alle cure mediche, all'intrattenimento, alla carta stampata, istruzione e sviluppo culturale è sempre lo Stato, attraverso una sofisticata rete di funzionari.

Nella giusta misura, tutti sono, in fin dei conti, funzionari dello Stato che, oltre che datore di lavoro, regola anche ciascuna delle istanze della vita umana nella misura della necessità di ogni cittadino. Anche la vita amorosa è quella consigliata e regolata da un organismo statale apposito, che stabilisce le norme per la formazione di coppie e per il riconoscimento del momento in cui il corteggiamento è sufficientemente maturo da poter sfociare nel matrimonio. L'informazione, in compenso, circola attraverso una rete di giornali mantenuta esclusivamente dallo Stato: un giornale nazionale principale, quelli regionali (uno per ogni provincia) e quelli locali (uno per ciascun comune).

Tra i comuni del paese, lo Stato rialloca le eccedenze in modo che nulla manchi a nessuna di queste e, conseguentemente, a nessuno dei suoi cittadini. L'egualitarismo, come si vede, si sviluppa simultaneamente nella rete di vite individuali e nella scacchiera spazializzata dei 1000 comuni raggruppati nelle 100 province che, infine, con precisione decimale, costituiscono la totalità dello Stato. La rappresentazione politica si ha, a livello nazionale, attraverso due deputati per comune, e il potere esecutivo si assottiglia a un centro decisionale costituito da 16 membri - che equivale al nucleo dei saggi che abbiamo già osservato nelle utopie tradizionali. Risiede qui, in questa spazialità rigorosamente matematica e in questa condivisione umana di ciascun tratto di vita comune, la conciliazione icariana tra l'uguaglianza sociale e l'uguaglianza matematica. 


\section{Cabet e il contesto storico dopo il successo di Icaria}

Volgiamoci ora verso il contesto storico di Cabet, creatore di questa ben organizzata utopia letteraria. Abbiamo osservato in precedenza come circa quattrocento ammiratori suoi e del suo romanzo utopico, tutti ispirati da ideali comunisti, abbiano manifetstato a un certo punto il desiderio di concretizzare il sogno icariano. Per discendere dai cieli dell'immaginazione letteraria alla terra delle realizzazioni possibili, il modello icariano esigeva che si trovasse un dittatore saggio, l'Icaro primordiale del romanzo Ma chi, se non lo stesso Cabet, avrebbe potuto assumere questa funzione? Tuttavia all'atto pratico lo scrittore francese si trasformò in un dittatore per nulla ammirato dalla piccola popolazione che confidava in lui e che con lui tentò una colonizzazione egualitarista nelle terre acquistate da una compagnia statunitense. Una volta installati in Illinois gli utopici pionieri e deciso a realizzare il suo sogno con l'incrollabile determinazione di un Icaro e con il pugno di ferro, Cabet inflisse ai suoi correligionari una rigorosa austerità icariana. Particolarmente impopolari furono le dure misure imposte alla comunità nel 1851, durante una crisi. Finì per vigilare sulla vita privata degli icariani, per proibire di bere e di fumare e persino per fomentare un sistema di vigilanza comunitaria nel quale tutti avrebbero dovuto spiare e tenere sotto osservazione tutti. Estremamente criticato, Cabet vide l'assemblea dei membri richiedere la sua espulsione nel 1856, poco prima della sua morte. Gli icariani proseguirono la loro esperienza per alcuni decenni, ma nel 1895 la comunità finì per sciogliersi. Essi si erano trasformati in comuni agricoltori e ora dichiaravano di essere semplici coloni, come tutti. L'esperienza utopica, dopo essere passata da una piccola svolta locale-distopica anche nel periodo dell'egemonia di Cabet, alla fine si trasformava in una piccola comunità come qualsiasi altra ${ }^{41}$.

L'esperienza di Cabet - e il suo tentativo di trasferire nella vita reale qualcosa della Icaria che aveva immaginato - mostra una fragilità comune a molte delle esperienze utopiche che tentarono di tradursi in realtà. L'attuazione dell'utopia, in tutti questi casi, avrebbe dovuto essere garantita dall'azione individuale dei grandi realizzatori. Poteva trattarsi di un dittatore-fondatore, di un comitato di saggi, di un industriale generoso, di un benevolo idealista che aveva bisogno di convincere nobili e importanti industriali per finanziare il suo appassionante progetto. L'utopia, in questo caso, non si sarebbe sviluppata a partire dalle insoddisfazioni e dalle domande sociali, ma

\footnotetext{
${ }^{41}$ Le imprese utopiche sul suolo statunitense, tra cui l'experienza icariana di Cabet, furono studiate da vari autori: NORDHOFF, Charles, The Communistic Societies of United States, New York, Harper \& Brothers, 1875; HINDS, William A., American Communities and Co-Operative Colonies, Chicago, Charles H. Kerr \& Co, 1908; HOLLOWAY, Mark. Heavens on Earth: Utopian Communities in America, 1680-1880, New York, Library Publishers, 1951; HINE, Robert V., California's Utopian Colonies, Berkeley, University of California Press, 1983; PITZER, Donald E., ed. America's Communal Utopias. Chapel Hill: University of North Carolina Press, 1997. Sull'esperienza icariana, si vedano specificamente: SHAW, Albert, op. cit.; SUTTON, Robert P., op. cit.; LARSEN, Dale R., op. cit.; WIEGENSTEIN, Steve, op. cit.
} 
avrebbe dovuto essere concessa all'umanità da volenterosi. Non era una conquista sociale ma un dono.

Come si nota, l'Icaria di Cabet - un'utopia letteraria che aveva rispondenze con altre che l'avevano preceduta - dialoga, almeno parzialmente, con i diversi modelli utopici menzionati in precedenza. Come questi - che vanno dalle utopie immaginate da Moro, Campanella e Bacon e che si rifacevano ai tentativi o alle speranze reali di concretizzazione delle società perfette, alle realizzazioni utopiste elaborate o pianificate da Fourier, Saint-Simon e Owen - anche l'Icaria fu importante per aver fatto discutere e ridiscutere la possibilità di estinguere l'oziosità e lo sfruttamento dell'uomo sull'uomo, oltre ad altre diverse tematiche pressanti tipiche del secolo del consolidamento della società industriale. Il cammino che Cabet e altri autori dell'immaginazione utopica preconizzavano, tuttavia, era "utopico" in un senso molto specifico - si trattava di un percorso ancorato all'idea per cui la riforma sociale, o persino la rivoluzione, avrebbe potuto essere condotta e finanziata da membri progressisti della borghesia nascente. In alcuni casi, troviamo un modello fondato sull'idea di una società egualitaria realizzata a partire dalla benevolenza liberale e diretta da un nucleo di saggi progressisti, come avvenne nel caso delle proposte che culminarono con le speranze di Fourier per la futura realizzazione dei suoi falansteri. In quest'ultimo caso specifico, il nucleo che avrebbe dovuto pianificare dettagliatamente il funzionamento della società, che finì per ridursi a Fourier stesso, si sarebbe costituito nell'esatto momento della nascita di questa. Ciò, almeno in parte, che era previsto tanto nell'utopia letteraria che nella società agricola reale che Cabet tentò di installare in Texas e in Illinois. In Saint-Simon, come già era avvenuto nella New Atlantis di Bacon e anche nella Città del Sole di Campanella, si palesava un'élite altruista di saggi che si dedicava incessantemente a studiare nuove maniere per migliorare la vita nella collettività.

Il sogno di sensibilizzare governanti e facoltosi capitalisti nei confronti di un grande progetto di radicale riforma sociale - fino a convincerli a investire in qualcosa che, in verità, avrebbe implicato la loro fine - mise in evidenza uno dei modelli egualitari più intriganti fra quelli che si svilupparono nel corso del XIX secolo. Owen, essendo un industriale di successo, poté provvedere da solo al ruolo complementare di sognatore e di investitore, ma la collettività-modello che tentò di impiantare in America fallì a sua volta, così come la più modesta proposta dell'Icaria americana di Cabet. Il Falansterio di Fourier e le Colletività di Owen e, a fianco di queste, i tentativi pionieristici di Cabet sul suolo americano, facevano parte di un sogno sufficientemente avanzato per il XIX secolo, ma la verità è che, per lo meno fino ai limiti della loro epoca, queste idee si rivelarono inefficaci. Sarebbero state possibili in un successivo futuro? 


\section{Considerazioni finali}

Come abbiamo potuto vedere nel corso di questo esame della società immaginaria realizzata dall'Icarie di Cabet, già divenuta un classico della letteratura utopista, e, marginalmente, anche dall'esame di alcune delle proposte egualitarie ed egualitariste prodotte nei secoli XVI e XIX, vi sono molti problemi e dilemmi connessi a un modello in grado di sistematizzare, in ossequio alla giustizia sociale, l'equilibrio tra lavoro e ozio, controllo e libertà, produzione e consumo - solamente per menzionare alcuni dei principali binomi evidenziabili nelle società industriali. Le proposte di rendere possibile una sistematizzazione più effettiva dell'organizzazione una società realmente egualitaria si dimostrarono lacunose I modelli di egualitarismo dei secoli XVII e XVIII toccarono solo marginalmente la questione dell'attuabilità del principio dell'uguaglianza durante il processo di distribuzione della ricchezza prodotta dalla società . "Uguaglianza tra chi" e "Uguaglianza di che cosa" - domande di fronte alle quali queste utopie e movimenti avevano risposto con la formula "uguaglianza di tutti in tutto" - sono necessarie ma non sufficienti per l'elaborazione sistematica di una riflessione approfondita sull'uguaglianza e l'adeguamento ad un modello di società.

Per la preservazione di una società egualitaria sarebbe stato necessario andare oltre il "conseguimento collettivo della ricchezza", fosse esso consensuale o raggiunto attraverso delle rivoluzioni. Sarebbe necessario discutere, certamente, la questione della "realizzazione della ricchezza" e della sua distribuzione per quelli che saranno i beneficiari dell'uguaglianza sociale. Alla riflessione sull'uguaglianza che deve sempre partire da due questioni originali ("uguaglianza tra chi" e "uguaglianza di che cosa"), sentiamo la necessità di aggiungere una terza domanda fondamentale, importante quanto le due precedenti. È infatti assecondando il paradigma proposto da Bobbio, discusso nel primo paragrafo, che sorge spontaneo un interrogativo, quando parliamo di uguaglianza: “Uguaglianza secondo quali criteri?”.

Ammettiamo che, perché si possa parlare in certi termini di egualitarismo, debbano essere stati stabiliti da un lato i beneficiari dell'uguaglianza (tutti o alcuni), dall'altro lato l'oggetto di azione egualizzatrice (uguaglianza di qualcosa - come i diritti politici o la libertà - o uguaglianza di tutto, come nelle proposte egualitarie più radicali). La questione da porre per una realizzazione duratura di una società egualitari sta verte sulle modalità con cui distribuire tra i beneficiari socializzazione ciò che verrà messo in comune. Così, particolarmente per le utopie o tentativi egualitari più radicali, uno dei problemi principali, fu sempre quello della distribuzione della ricchezza. Il modello egualitario di Buonarroti - militante rivoluzionario che è stato menzionato solo di sfuggita in questo articolo - poggiava, ad esempio, sulla prospettiva romantica per cui «quando non ci sono palazzi non ci sono neppure capanne», cosicché l'uguaglianza finale sarebbe sbocciata più o meno spontaneamente da un unico gesto. La Cuccagna medioevale, terra immaginaria dove tutto ciò che era necessario nella vita sorgeva spontaneamente, si sosteneva su una filosofia dell'abbondanza che 
non necessitava di pianificare rigorosamente il sistema lavorativo. Nel frattempo i modelli egualitari proposti dal socialismo scientifico a partire da Marx e Engels (1880) - modelli la cui discussione va oltre le possibilità di questo articolo- dovettero affrontare queste questioni in modo più concreto e, tuttavia, vennero enfatizzate formule più specifiche di distribuzione della ricchezza ("A ciascuno secondo la propria necessità" o "a ciascuno secondo la propria produzione"). Fu in questo senso che i pensatori politici interessati ad un egualitarismo più moderno, facendo leva su interessi maggiormente pragmatici, dovettero tornare a dialogare con le idee che già contenute in vecchie discussioni filosofiche e con i concetti di uguaglianza aritmetica o geometrica proposti da Aristotele. Questa discussione, tuttavia, ci invita ad una riflessione più specifica,

Le opere della letteratura utopica e distopica, d'altra parte, sono ugualmente importanti per mettere in discussione l'uguaglianza e la realizzabilità o l'irrealizzabilità dei modelli immaginari che cerchino la giustizia sociale. Studi sulle utopie letterarie - simili a quella prodotta da autori come Cabet ancora nel XIX secolo - possono contribuire a mantenere sempre attuale questa discussione vitale sulla ricerca umana dell'uguaglianza sociale. Immaginare l'uguaglianza, si può aggiungere, è un primo passo per schiudere la possibilità della sua realizzazione futura. 


\section{L'AUTORE}

José D'Assunção BARRos è professore associato presso l'Universidade Federal Rural do Rio de Janeiro. ProfessorColaborador nel corso di dottorato in História Comparada della Universidade Federal do Rio de Janeiro, si è addottorato in Storia presso l'Universidade Federal Fluminense.

URL: < http://www.studistorici.com/progett/autori/\#Barros > 